
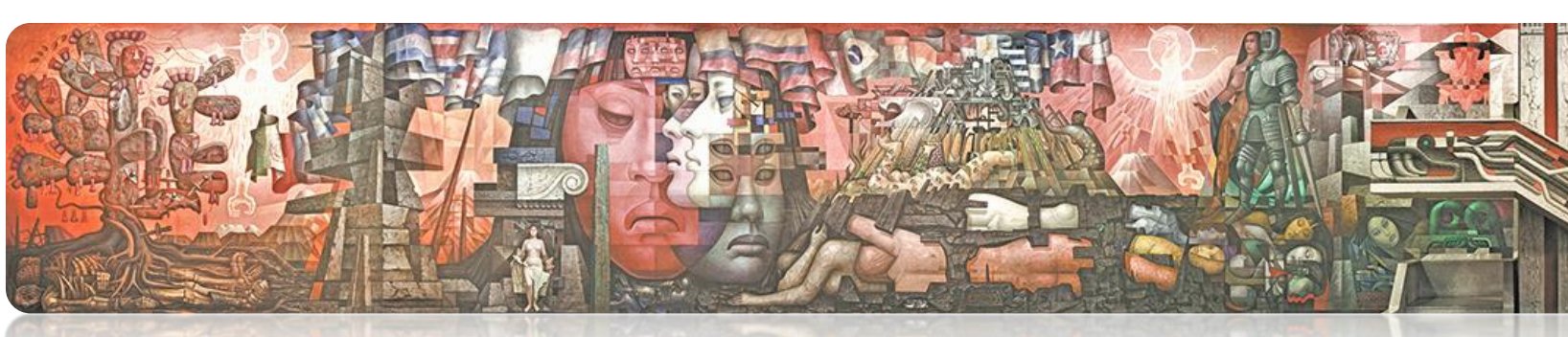

\title{
En la Ruta republicana de O’Higgins y San Martin: la configuración de Valparaíso como un espacio geo-histórico en la primera mitad del siglo XIX
}
On the Republican Route of O'Higgins and San Martin: the configuration of Valparaíso as a geo-historical space in the first half of the 19th century

Jaime Vito Paredes*

\section{RESUMEN}

Estudiaremos el proceso histórico de configuración de Valparaíso como un puerto importante en el Pacífico sur, observando como en sus diversas etapas se va estratificando un espacio político y económico que, condicionado por las regiones de los valles interiores, se irá transformando luego en un espacio de conexión con el Mundo del Pacifico sur y el Mundo Atlántico de acuerdo con los cambios operados a comienzos del siglo XIX. En ese trayecto se encontrará con el trazado de la ruta de O'Higgins y San Martín y experimentará un involucramiento que lo comprometerá con el destino republicano de todo el Pacífico sur durante la primera parte del siglo XIX.

Palabras claves: Espacio geo-histórico, política-económica, Ruta republicana, Mundo del Pacífico sur, Unión Americana, el Acontecimiento de la Ruta.

\begin{abstract}
We will study the historical process of the configuration of Valparaíso as an important port in the South Pacific, observing how in its different stages a political and economic space is stratified which, conditioned by the regions of the interior valleys, will later be transformed into a space of connection with the South Pacific World and the Atlantic World in accordance with the changes that took place at the beginning of the 19th century. In this journey, it will meet with O'Higgins and San Martin's route and will experience an involvement committed to the republican destiny of the entire South Pacific during the first part of the 19th century.
\end{abstract}

\footnotetext{
* Pontificia Universidad Católica de Valparaíso, Doctor en Historia. ORCID: https://orcid.org/0000-0002-1059-0054. Correo electrónico: jaimepvitop@yahoo.es.
} 
Keywords: Geo-historical political-economic space, republican route, South pacific world, American Union, the Route event

Recibido: septiembre 2021

Aceptado: diciembre 2021

\section{Introducción: El republicanismo que antecede al trazado de la ruta de O’Higgins y San Martín}

Según lo consigna el historiador Diego Barros Arana, en medio de los inicios de la cristalización del nuevo orden independiente en Chile, José de San Martín cruzaba la cordillera desde las provincias del Río de la Plata para preparar la expedición sobre el Perú, trayendo consigo las bases de un plan político para dar a estos países un gobierno que creía el único estable y adecuado a la situación ${ }^{1}$. En el devenir de los acontecimientos, la empresa de liberación del Perú de manos de las fuerzas Realistas debía ser articulada en conjunto tanto por Chile como por las Provincias del Río de la Plata, pero, debido a las dificultades políticas observadas en el lado oriente de la cordillera de los Andes, recaería sobre Chile el papel y la responsabilidad de organizar y financiar la expedición libertadora. Estamos en los años 1819-1820, en que la transición entre el antiguo y el nuevo orden aún no se divisaba con claridad y más bien se avizoraba como un estadio tambaleante y movedizo que no lograba desenraizarse de la etapa colonial. Los contemporáneos y especialmente los líderes del movimiento independentista, imbuidos en una cultura política más o menos ilustrada, daban por sentada la transición y auguraban que era cuestión de tiempo que el cambio epocal se produjera. Sin embargo, hombres como Bolívar, Sucre, O’Higgins y San Martín, ya desde 1815 (y antes) pensaban que la realidad hispanoamericana y su destino debía observarse y sopesarse con más calma y racionalidad, pues, si bien el objetivo de instaurar repúblicas era deseado por todos, el entusiasmo y la adhesión a la noble causa habían conducido, en los primeros esfuerzos, a construir republicas aéreas que rápidamente se derrumbaron con la reconquista de los ejércitos monárquicos ${ }^{2}$. De este modo, en los estrategas de la independencia existió, en el camino más práctico a seguir, un antes y un después de 1815, para llegar a dibujar en el espacio republicano las relaciones políticas. La consolidación se irá trazando por partes y en este

\footnotetext{
${ }^{1}$ Barros Arana, Diego.1892. Historia general de Chile, Tomo XII, Rafael Jover Editor, Santiago de Chile, pp. 5 y ss.

2 Ver Bolívar, Simón.1986. Carta de Jamaica. Contestación de un americano meridional a un caballero de esta isla (Henri Cullen), Ideas en torno de Latinoamérica México, Tomo I, UNAM, p.429. Las advertencias sobre las repúblicas aéreas recuerdan que, la república, como una noción que se encarna en un léxico, sólo aterriza en un espacio histórico bien asentado si se considera la relación entre el alcance universal del concepto y sus implementaciones históricas y las condiciones regionales-continentales y locales para el cultivo de su espíritu en un cuerpo social.
} 
camino, Valparaíso, como espacio local, en la constitución de un eje con Santiago, constituirá una de esas partes y jugará como territorio, un punto de partida y de llegada de las coordenadas de una cartografía republicana regional-continental durante la primera parte del siglo XIX. La expedición libertadora se enmarcará en esta estrategia y buscará cerrar un primer ciclo de la independencia que se había abierto en 1806 con la invasión inglesa de Buenos Aires y se cerraría en 1821-1824 con la derrota de las tropas españolas en el cono sur americano.

Cuando hablamos de republicanismo pensamos en un concepto que desplegó su propia historia desde el siglo XVIII hasta los inicios del siglo XIX en el Mundo Atlántico y luego en los siguientes 200 años. Con la perspectiva temporal, hoy tendemos a desagregar aspectos del concepto que se han ido encriptando a través del tiempo, pero que es necesario distinguir pues los referentes discursivos que han circulado en el mundo atlántico y en occidente y que le han servido de soporte, no siempre se cristalizarán en referenciales históricos que reflejarán, como el rostro en un espejo, la realización factual de los mismos. Ya lo decía Claudio Veliz al caracterizar el orden político hispanoamericano surgido desde la independencia: más allá de las banderas del liberalismo y la división de los poderes, lo que observamos es una continuidad en la tradición centralista lo que marcaría las diferencias políticas y de civilización entre los dos mundos del nuevo mundo ${ }^{3}$. De ahí que el estudio sobre el republicanismo existente sobre la época del inicio de la ruta O'Higgins-San Martin, considere cada vez más la diversidad semántica que adquiere el concepto según sea la región de que se trata y del modo como se territorializa en un proceso de adaptación que conjuga diversas variables ${ }^{4}$.

En este sentido, cuando nos acercamos al caso de Chile en la época de inicio de la Ruta, estamos observando el cierre de un primer momento republicano y nos situamos ad portas de un segundo momento que no se dará sin estabilizar el espacio republicano en términos continentales. De ahí la importancia de consolidar ciertos nodos en esta red que intenta desplegar el nuevo orden, siendo uno de estos el de Valparaíso (en estricto sentido ValparaísoSantiago) que requerirá de su propia consolidación interna y también de una inserción en un campo internacional en el que la república en la que se inscribe pueda ser reconocida en su soberanía. En esta línea podemos señalar que el republicanismo, ante los peligros que le acechaban, no solo debería tomar distancia de la monarquía absoluta y de los intentos por instalar una monarquía constitucional (siguiendo el ejemplo de Brasil), sino también de los intentos más utopistas de los primeros años que intentaron darle forma a una república federal

\footnotetext{
${ }^{3}$ Véliz, Claudio.2011. Los dos mundos del Nuevo mundo. Cultura y economía en Angloamérica e Hispanoamérica, Ediciones Tajamar, Santiago, passim.

${ }^{4}$ Para el estudio del concepto de republicanismo y del movimiento que generó en el Rio de la Plata y en Chile durante la primera mitad del siglo XIX Cr. Fernández, Sebastián y Vito, Jaime (2017). "El republicanismo en las Provincias Unidas del Rio de La Palta y Chile en la primera mitad del siglo XIX", en: Cicerchia, Ricardo (compilador), Región y naciones. Instituciones, ciudadanía y performances sociales. Chile y Argentina (siglos XIX-XX). De local a lo Global, Buenos Aires, Editorial Prohistoria ediciones y PEI Sur-PUCV, pp.23 - 68.
} 
o a un federalismo de Estado al estilo norteamericano. Entre los múltiples ensayos y errores experimentados desde el inicio del proceso de la independencia, se ha llegado al cierre de un primer momento republicano alrededor de 1820, en el que la necesidad de consolidar una nueva legitimidad y un nuevo ordenamiento que implicaba el reconocimiento de la autoridad del Estado y del gobierno, se trasformó en una imposición no exenta de autoritarismo. La forma monárquica constitucional fue muy difícil de incorporar en la nueva legitimidad no sólo porque la independencia se había hecho- con una guerra de por medio- en contra de otra forma de monarquía (absoluta) sino también por la casi imposible tarea de construir una nueva casa real en medio de profundas diferencias a partir de oligarquías que no eran homogéneas ni adscribían en sus inicios a una idea de identidad nacional. De ahí que la estabilización del espacio republicano pasaba por lograr asir en un sólo eje la multiplicidad de cuerdas que expresaba lo heterogéneo de una realidad socio cultural a la cual se le estaba derrumbando la antigua matriz política.

Ahora bien, existe también una relación estrecha de dependencia mutua entre la construcción del Estado-Nación y el despliegue de un espacio público republicano, pues si el republicanismo requiere la elección periódica o regular de los cargos que ofician como conductores de los poderes del Estado para afirmar su autenticidad y su legitimidad, este procedimiento no se puede realizar sin la existencia aunque sea limitada de un espacio público que certifique los actos y en el cual estos actos puedan ser visibles en toda su positividad. Y, hasta el momento en que se inicia el recorrido de la Ruta emancipadora, el concepto de espacio público también ha atravesado por cambios importantes en el mundo atlántico en la transición al siglo XIX. En efecto, este ya no se relacionará sólo con una dimensión política circunscrita territorialmente a las ciudades y al espacio en el que se encuentran el Estado y la sociedad civil, en un redondel de observaciones y controles mutuos asentado en un modelo jurídico, sino que ahora se asociará con otro eje que cruzará dos definiciones de lo público y que se enlazarán cual helicoide que dará por resultado una resignificación de aquello que se venía entendiendo por espacio público ${ }^{5}$. Esta dimensión que irrumpe con fuerza en el contexto de transformación de la economía-mundo Atlántico hacia un sistema mundo, es la del espacio económico. En la época de los inicios de la Ruta, se tiene la urgencia de estabilizar el espacio republicano, pues, el Estado simultáneamente requerirá insertarse en las redes económicas del sistema mundo dominadas ahora por las economías industriales, para poder financiarse y generar un mercado

\footnotetext{
${ }^{5}$ Para el tema de la configuración del espacio público y la distinción de las dimensiones que lo componen al entrelazarse una lógica jurídica del modelo ilustrado del siglo XVIII con una lógica disciplinaria que comienza a incluir a las lógicas económicas de un sistema mundo de Ira revolución industrial cf. Vito, Jaime.2018. Capítulo Problematizando la definición del espacio público moderno: Un acercamiento desde una mirada historiográfica, en Eduardo Cavieres Fernández y Eduardo Cavieres Figueroa (editores), Tensiones entre Estado y Ciudadanía. Repensando espacios, significaciones y tiempos, Valparaíso, PEI-SUR- PUCV y CEA Centro de Estudios Avanzados de la Universidad de Playa Ancha, Ediciones de la Editorial de la Universidad de Playa Ancha, pp. 31 - 46.
} 
propiamente nacional. En este sentido, Valparaíso resulta finalmente transformado en la salida de Santiago hacia las redes de intercambio de la economía atlántica y por ende, el espacio republicano completará su propio rostro con un mercado nacional en construcción que requiere conectarse a dichas redes.

\section{La configuración del espacio geo-histórico de Valparaíso. De caleta marginal a puerto principal del nuevo Estado Chileno}

Siguiendo nuestra hipótesis, en la época en que se organiza la expedición libertadora del Perú que parte en su etapa marítima en Valparaíso, el puerto ha comenzado un ciclo de crecimiento urbano y comercial y una dinámica de movimiento de mercancías y de personas que nos hablará de una conexión muy estrecha con la actividad comercial del Pacífico sur, pero también con el espacio que se ha transformado en el centro-capital del nuevo Estado republicano al cual estará ind efectiblemente unida en la configuración del nuevo orden. Pero, ¿cómo se constituye este nuevo eje Santiago-Valparaíso? ¿Es una configuración regional geo-histórica o no siéndolo en estricto sentido, las relaciones políticas, los intereses económicos y las tensiones regionales al interior del nuevo Chile explicarán finalmente que Valparaíso se constituya por largo tiempo en el principal puerto de Santiago y de la República? ¿Es esta circunstancia configuradora del papel de Valparaíso en la ruta de la independencia del Perú lo que nos permite pensarlo como uno de los nodos claves para explicarnos su protagonismo en la configuración de un espacio republicano en el cono sur americano? En verdad Valparaíso tendrá un protagonismo no sólo en el primer ciclo que se cierra, de consolidación del orden republicano en el Pacífico sur, sino también lo tendrá en un segundo ciclo que tenderá a cerrarse en los años 1860 del siglo XIX. ¿De qué modo una problemática continental y atlántica como es la cuestión de republicanismo que se juega en el Pacífico sur tendrá en esta pequeña localidad uno de sus nodos comunicantes? En este punto es necesario ralentizar nuestra marcha y detenernos atentamente en este nodo y en sus especificaciones particulares, en el modo como este cobrará protagonismo en la zona central de la costa chilena y de qué manera históricamente no nos encontraremos con el descubrimiento de una profundidad histórica encapsulada y que simplemente había que develar, sino con la historia de una ciudad que se fue haciendo en las circunstancias, que en cada coyuntura tuvo que resolver tensiones y direcciones alternativas de salida. Son tres los momentos que identificamos en la Historia de Valparaíso en los que opera esta tensión integral de direcciones alternativas que condicionarán una reorganización necesaria de carácter local (el puerto, la ciudad, la región) y que también lo insertarán y lo vincularán con conexiones internacionales: uno, los antecedentes coloniales: Del Valle de Quintil a Valparaíso. El papel adjetivo de Valparaíso entre el valle de Aconcagua y el valle de 
Santiago antes del siglo XIX ${ }^{6}$; dos, Valparaíso en la época de O'Higgins y el inicio de la ruta O’Higgins-San Martín. El papel sustantivo de Valparaíso en la instauración del espacio republicano chileno y del Pacifico sur; tres, Valparaíso como "entrepot" en la primera mitad del siglo XIX: mirando hacia el Pacifico sur y más allá. Por razones del contenido central de este trabajo, sólo nos detenemos en el segundo momento.

Como sabemos, los circuitos comerciales y la circulación de riquezas, bienes y personas, han operado desde siempre como lazos conectores de mercados, ciudades y regiones, muchas veces atravesadas por soberanías políticas muy diversas y a menudo opuestas. En un sentido más profundo $y$, en duraciones más prolongadas, las conexiones terminan no pocas veces transformándose en integraciones sociales, culturales y políticas. En relación con el papel de la región de Valparaíso-Aconcagua y su implicancia en la génesis de la nación chilena en la transición del siglo XVIII al XIX y luego en el papel jugado en la consolidación del espacio republicano del Pacífico sur, cabría preguntarse por los resultados de la entrada en vigencia del decreto de libre comercio de 1778 y del decreto de libre comercio chileno-republicano de 1811, ambos inspirados en la libertad de comercio, pero diferenciados en el sentido de que el decreto del naciente Estado-nación intentaba reorientar las conexiones de los circuitos dejando, tal como ocurrió en el conjunto del continente hispanoamericano, el interés por los mercados americanos en desmedro del mercado Atlántico representado por Inglaterra.

En este marco histórico, Valparaíso-ciudad fue transformándose en un centro cada vez más gravitante en la redireccionalidad de los circuitos comerciales, llegando a conformar un verdadero entrepot, según la tesis clásica de Jacqueline Garreaud ${ }^{7}$. La incorporación al mercado capitalista atlántico, aquel de la primera revolución industrial, implicó un efecto desequilibrante para el conjunto de las regiones chilenas que, a su vez, se incorporaban al nuevo orden republicano. La transición al siglo XIX fue también auspiciosa para Valparaíso en el plano poblacional y demográfico. Resuenan aquí los ecos de la caracterización que Vicuña Mackenna hacía sobre el puerto en el sentido de que los tiempos difíciles, los de la guerra, por ejemplo, eran los acicates más potentes para que esta ciudad resurgiera del letargo en el que usualmente caía. Desde 1779 a 1813 Valparaíso creció de 2.151 habitantes a 5.317, duplicándose la población con un crecimiento anual del $4.5 \%$, advirtiéndose que este crecimiento se dio más en el sector semi-urbano del barrio Almendral, aquel que se encuentra

\footnotetext{
${ }^{6}$ Algunos de estos desarrollos los hemos trabajado en Cavieres, Eduardo y Vito, Jaime.2020. "Región y nación: Valparaíso- Aconcagua y la configuración de una región en el naciente Chile republicano", en Armando Cartes Montory (Editor) Región y Nación. La construcción provincial de Chile Siglo XIX, Editorial Universitaria, Santiago de Chile, pp. 163 - 185.

7 Garreaud, Jacqueline. 1994. "La formación de un mercado de tránsito, Valparaíso: 1817-1848”, en Revista Nueva Historia, Año 3, №11, Londres, pp. 157 - 194.
} 
más cerca de la entrada por tierra a Valparaíso, que en el sector del barrio principal" ${ }^{8}$. En los últimos años del siglo XVIII y primeros del siglo XIX, la migración desde el interior a la costa comenzaba a producir importantes cambios demográficos con sus consecuentes efectos sociales, económicos y políticos.

También observamos la formación de una incipiente clase de empresarios de origen extranjero de tal modo que, "para la década de 1820 el descenso de los españoles en la actividad comercial fue muy violento y por el contrario la emergencia de los comerciantes británicos fue también espectacular marcando un hito importante en el curso de la economía mundial y la incorporación de nuestro medio a ella" ${ }^{\prime}$. Hay que destacar que el impacto del crecimiento demográfico fue no sólo cuantitativo sino también cualitativo pues no sólo era gente mayoritariamente joven sino con propósitos de vincularse a la complejidad de la vida comercial que se modernizaba en el sentido capitalista. El Viajero inglés Samuel Haigh ha dejado su testimonio para la época (1820):

"el muelle de Valparaíso, tiene el fuerte a un costado y en él están los departamentos del gobernador. Allí está también el mercado donde se expende carne de toda especie (salvo de ternera), aves de corral, caza, vegetales y abundante fruta venida del hermoso valle de Quillota, que es considerado como el más rico de todos en la provincia entera. El precio de la vida era muy barato, pero debido a la afluencia de extranjeros, su costo ha crecido considerablemente, porque es observado que donde los ingleses hacen su aparición, tienen la peculiaridad de alzar el precio de los víveres, pues pagan lo que se les pide; esto sin mencionar las liberalidades adicionales que con frecuencia acuerdan...la población de Valparaíso se estimaba entonces en cerca de seis mil habitantes, pero actualmente ha crecido hasta bordear el doble de ese número..."10.

El mismo Haigh refiere en su relato que en 1817 Valparaíso presentaba un rostro muy distinto al que observó en 1821. De hecho, en aquel año y según su testimonio, solo residían ahí dos ingleses, en cambio, en su último viaje encuentra a dos mil, que ya conforman una verdadera colonia extranjera dedicada fundamentalmente al comercio y a las incipientes finanzas.

Diremos que, a partir de 1820 la ciudad comienza a transformarse en un eje comercial y naviero que consolida su conexión con el interior de la región, especialmente con el valle de

\footnotetext{
8 Salinas, René. 1971. "Caracteres Generales de la evolución demográfica de un centro urbano chileno: Valparaíso, 1685-1830", Santiago de Chile, Revista Historia 10, Santiago, pp.178.

${ }^{9}$ Estrada, Baldomero. 2012. "Desarrollo empresarial urbano e inmigración europea: españoles en Valparaíso 18801940", Tesis Doctoral Universidad Complutense, Madrid, especialmente el capítulo introductorio al siglo XIX, p.18 ${ }^{10}$ Haigh, Samuel. 1917. Viaje a Chile durante la época de la independencia, Santiago de Chile, Colección de autores extranjeros relativos a Chile, Chile, Imprenta Universitaria, pp. 62 - 63.
} 
Quillota, que se subordina cada vez más a la costa. Luego, el puerto, en el transcurso del mismo decenio de los años 1820, incrementará su rol de imán del comercio exterior que lo subordinará al interés del Estado-nación y al de su centro en la capital de Santiago de Chile. Se hace necesario extender una serie de reglamentaciones y medidas con el objeto de regular, según el interés del Estado, los efectos positivos de la actividad económica para las necesitadas arcas fiscales del país. Junto al reglamento promulgado en 1820, que contenía disposiciones relativas al depósito de mercancías en tránsito, las medidas se complementan con disposiciones promulgadas en 1822 y en 1823, relativas a la instalación de bodegas privadas y depósitos flotantes y almacenes de propiedad del gobierno, lo que venía a regular la condición de puerto de almacenaje y de paso de Valparaíso, instituyéndose la idea de puerto eje en el Pacífico sur americano, que funcionaba también como nodo de distribución de mercaderías que venían de puertos europeos, del Atlántico sur y que pasaban hacia lugares tan lejanos como la Polinesia o la Micronesia, hacia la otra orilla del océano Pacífico. Todo este movimiento económico transformará a Valparaíso en un puerto importante en el Pacífico sur, lo que de algún modo hizo que las turbulencias políticas del país durante la década de 1820 tuvieran en esta parte del territorio costero de la región, efectos menos dramáticos.

\section{Valparaíso, punto de partida marítimo de La expedición libertadora}

En este escenario y desde esta ciudad es donde la expedición libertadora del Perú partirá un día de 1821. Y esta partida será el resultado de una compleja preparación que debió librar batallas burocráticas, sorteando una larga serie de obstáculos en la que se entremezclaban situaciones personales y asuntos de Estado. La preparación de la expedición libertadora del Perú era torpedeada fundamentalmente por las tensiones existentes entre las nuevas regiones que se incorporaban a la independencia como por cierta desidia a la cual se abandonaban algunos dirigentes, pensando que los realistas de Lima estaban ya derrotados y era cuestión de tiempo que el espacio republicano se afirmara desde el Río de la Plata hasta Venezuela, pasando por Lima y Quito. Diego Barros Arana afirmará que el Congreso nacional de las provincias del Río de la Plata reunido en Buenos Aires y que celebraba en la persona de José de San Martin la derrota de los realistas en Chacabuco y Maipú, no le escatimaba elogios junto a una ciudad que se contagiaba con la atmósfera de fiesta y de celebración: "el vulgo estaba enternecido. Cuando el general (San Martin) volvió acompañado del excelentísimo señor director, siendo entonces más de uno el objeto de la admiración y del reconocimiento público, pareció el pueblo absorto en la contemplación de su propia grandeza...estas manifestaciones de carácter oficial fueron acompañadas y seguidas de fiestas particulares con que algunos de los más distinguidos vecinos de la ciudad quisieron obsequiar a San Martín"11. Sin embargo, el

${ }^{11}$ Barros Arana, Diego. 1892. Historia general de Chile, Tomo XII, Editor Rafael Jover, Santiago de Chile, p.8 
mismo San Martin era consciente de las dificultades que se estaban presentando y también de las que se avecinaban con las divisiones emergentes en las diversas zonas de las Provincias Unidas del Río de la Plata donde la llamada anarquía expresada fundamentalmente en caudillismos locales y regionales no terminaba de sacudir el tinglado estatal que se pretendía instaurar. Extendiéndose por Entre Ríos, Santa fe y Córdoba las perturbaciones del orden no parecían cesar y esto colocaba un gran signo de interrogación sobre la realización efectiva de la empresa libertadora, pues una de las grandes preocupaciones de San Martín tenía que ver con el financiamiento y en las condiciones políticas vividas por las Provincias Unidas, este financiamiento era muy difícil de conseguir. San Martín tendrá en este sentido una disposición y voluntad de acero en términos de conseguir los recursos y en términos de implementar, según su propia mirada, el trayecto a seguir en la ruta hacia Lima pasando por Valparaíso-Santiago. Las negociaciones con Pueyrredón con quien le unían ciertas conexiones sociales y en especial la pertenencia a la sociabilidad masónica, se llevaron a cabo en las inmediaciones de Buenos Aires. Pueyrredón tenía en mente su propia ruta, donde un contingente de 5.000 hombres recorrería un trayecto que los llevaría al sur del Perú como una antesala antes de llegar a Lima. El enfrentamiento con el centro virreinal resultaría en lo inmediato muy costoso y de resultado incierto, por lo que era necesario evitar las pérdidas humanas y de recursos materiales. En esta línea de estrategia y de táctica militar, San Martín estaba convencido que la escuadra debería dirigirse al Callao y de ahí al asalto de Lima, aunque, naturalmente esta línea exigiría de mayores recursos y de mayor cantidad de tropas. Al final San Martín consigue una cantidad de 500.000 pesos de la época que se obtendrían de un empréstito interior y que se había decretado antes. Sin resolver acuerdo alguno sobre la estrategia militar a seguir y que en un consenso tácito se resolvería según las circunstancias, José de San Martín se dirigió a Mendoza con la intención de cruzar por la cordillera a Chile en pleno invierno con la urgencia de activar los preparativos de la ruta. Este cruce en sí no careció de dificultades, pues, intentó dos veces cruzar la cordillera y sin embargo debido a las nevazones, no era posible avizorar sendero alguno por el cual circular, lo que lo hacía cada vez devolverse a Mendoza, acerando su paciencia. Allí recibía noticias tanto de Chile como de Buenos Aires y muy pronto se encontrará con que los presupuestos de la expedición estaban cayendo, pues según Pueyrredón, fue imposible obtener dicha cantidad por los problemas económicos y la escasez de metal circulante. La cuota se redujo a un tercio, trastocando sensiblemente la empresa. Según el relato de Barros Arana: "San Martín...estuvo a punto de abandonar (la entereza de su carácter) en estos momentos en que la pobreza del erario, o más propiamente por la falta de una suma de dinero que según él era indispensable procurarse, iban a fracasar los planes tan pacientemente elaborados y de cuya realización dependía el afianzamiento de la independencia"12.

12 Barros rana, Diego. 1892. Ibídem., p.19. 
El general comenzó a elevar el tono de su correspondencia con Pueyrredón señalándole que, si no se recibía el financiamiento solicitado, el ejército reunido no sólo no podría cumplir con su objetivo, sino que estaba totalmente expuesto a su disolución. El 26 de septiembre le estampa su renuncia al mando del ejército, sea que este gesto haya sido expresión de su estado de ánimo o que con este acto quisiera tensionar al máximo la consecución de sus planes ${ }^{13}$. La preocupación prendió tanto en Chile como en el Río de la Plata, de tal forma que el agente chileno en Bueno Aires, Miguel Zañartu subrayó que su gobierno haría todos los sacrificios posibles para llevar a buen puerto la empresa libertadora y que como un esfuerzo conjunto, Chile y las Provincias Unidas del Río de la Plata debían nivelar sus gastos en igualdad de condiciones. De este modo Pueyrredón, que estaba alarmado por las consecuencias que traería la renuncia de San Martin tanto para la estabilidad interna como para el aseguramiento de la estabilidad republicana en el cono sur de América, prometió a San Martin que se cumpliría con lo acordado. Cuando por fin el general llega a Santiago el 29 de octubre, se encuentra en lo inmediato con una acogida festiva y con el general O'Higgins dado a la tarea de reunir tropas y conseguir fondos de financiamiento para la expedición. Hacia fines de ese año,

"el ejército debía componerse de 5.400 infantes, 400 artilleros, 200 jinetes y 100 zapadores, formando así un total de 6.100 hombres. El tren de artillería debía contar de 24 piezas de campaña con quinientos tiros para cada uno. Además del armamento correspondiente a cada soldado, se llevarían diez cañones de repuesto, tres mil fusiles y mil carabinas para armar nuevas tropas, con cuyo objeto se organizaría un cuerpo de oficiales, sargentos y cabos. El estado detallaba las municiones, las fornituras, los artículos de maestranza y de hospital militar y pedía tres meses de víveres para el ejército y la tripulación de los buques. Todo esto exigía un caudal considerable; pero San Martín agregaba, además, que era indispensable fletar buques mercantes para el transporte del ejército y llevar 200.000 pesos en dinero" ${ }^{14}$.

La organización de la expedición siguió sumando dificultades, pues entre los años 1819 y 1820 diversos eventos político-militares amenazaban el sur de Chile y diversos lugares del cono sur americano. Todo hacía pensar que la expedición nunca zarparía en condiciones ideales y que, en gran parte, el incierto destino era amortiguado por la capacidad de estrategas militares de San Martín y O'Higgins y el conocimiento y la experiencia de hombres como Lord Cochrane que se sumaban a la aventura de liberar a América de la monarquía española. En el diario de este último se subrayarán estas dificultades: "el gobierno no tenía crédito en tanto que su tesoro estaba exhausto por los esfuerzos hechos para organizar un ejército. Negociar un

\footnotetext{
13 Barros Rana, Diego .1892. Ibídem. p.19.

${ }^{14}$ Barros Arana, Diego. 1892. Ibídem, p.55.
} 
empréstito era imposible porque ya había sido negado. Merced a la influencia que yo tenía con los comerciantes ingleses, había conseguido de ellos un buen acopio de pertrechos navales y militares que contribuyesen a una suscripción que al efecto se había abierto" ${ }^{15}$. El almirante señalaba además que una de las tantas dificultades para embarcar tropas decía relación con los marineros extranjeros, quienes disgustados por la mala fe de quienes debían redituar el pago de salarios y otros beneficios, amenazaban con no embarcar. Ello obligó a que el propio lord Cochrane interviniera en persona prometiendo que una vez entrado en Lima se pagarían con puntualidad todos los atrasos devengados a cada uno de los marineros extranjeros que se alistaron voluntariamente en el servicio de Chile, dando a cada uno de los individuos según su clase, la paga entera de un año ${ }^{16}$.

Por fin, llegando los días 18 y 19 de Agosto de 1820 y mientras se resolvían los últimos nudos de la organización de la empresa, las tropas comenzaron a embarcar en Valparaíso, acompañados por bandas militares, lo que llevó a la población a volcarse a los muelles, quedando terminado el embarque de todo el ejército la tarde del 19 de agosto de $1820^{17}$. Como lo consignara una testigo excepcional, la viajera María Graham, O'Higgins y San Martín se preocuparon de proclamar los objetivos de la expedición y las consecuencias que traería para la convivencia entre los noveles Estados, incluida la futura república de Perú. En las proclamas que también se habían repartido previamente en el Perú, O’Higgins decía:

"no penséis que pretendamos trataros como a un pueblo conquistado; tal intento sólo puede caber en la cabeza de los enemigos de nuestra común felicidad. Nosotros aspiramos solamente a veros libres y felices: vosotros organizareis vuestro gobierno, escogiendo la forma que guarde armonía con vuestros hábitos, vuestra situación y vuestros anhelos; vosotros seréis vuestros propios legisladores, y por consiguiente instituiréis una nación tan libre como la nuestra"18.

San Martín, por otro lado, se unirá también al coro independentista: "la victoria tendrá por resultado que la capital del Perú vea por primera vez unidos a sus hijos, eligiendo libremente su gobierno y presentándose a la faz del mundo en la categoría de las naciones" ${ }^{19}$. Según la cronista inglesa, de este espíritu participaba también su compatriota Lord Cochrane, aunque San Martín manejaba discretamente los detalles del plan, creando con ello naturalmente cierta distancia. Según nuestra autora, el 21 de agosto de 1821, reinaba en la ciudad y en los protagonistas el mayor de los ánimos, cuando San Martín izó la insignia de capitán general al

\footnotetext{
${ }^{15}$ Lord Cochrane. 1863. Conde de Dundonald, Memorias, Imprenta José Masías, Lima, p.88.

16 lbídem, p.89.

17 Barros Arana, Diego. 1892. Ibídem, p.657.

${ }^{18}$ Graham, María. 1822. Diario de su residencia en Chile (1822) y de su viaje a Brasil, Madrid, Ed. América, , p.98.

19 Ibídem, p. 99.
} 
embarcarse en el buque de la capitanía general acompañado de los soldados de Chacabuco y de Maipú, que continuaban por el Pacifico la ruta esta vez hacia el norte. La escuadra, una vez alejada de Valparaíso, se detuvo en Coquimbo para reunir provisiones y embarcar las tropas reunidas en esa ciudad. Simultáneamente el director supremo Bernardo O'Higgins declaraba el bloqueo de todos los puertos situados entre los $2^{\circ}, 12^{\prime}$ y los $21^{\circ} 48^{\prime}$ de latitud sur, y extendiéndose, por lo tanto, el mismo entre Iquique y Guayaquil. Para cerrar simbólicamente este primer paso destinado a establecer el republicanismo en el Pacifico Sur, O’Higgins publicó un manifiesto el 31 de agosto de 1820 donde consignó "educado en el libre país de Inglaterra, se ha fortalecido en mi ese innato deseo de independencia que alienta en todo hombre nacido en tierra Araucana. Amante de la libertad, por principio y por sentimientos, juro ayudar a obtener la de mi patria o sepultarme entre su ruina" ${ }^{20}$.

\section{El republicanismo del Pacifico Sur americano después de O’Higgins y San Martin: La continuidad de la ruta en la Unión Americana.}

Desgraciadamente, estas grandes expectativas para el proceso de emancipación y para las futuras relaciones de Chile con el Perú, se debilitaron rápidamente y, en ello, el espacio geoeconómico y político de Valparaíso se transformará rápidamente en competencia Valparaíso-Callao.

La unidad americana en torno al republicanismo de los nuevos Estados-naciones pasó después de 1824 por ciclos que acercaban o alejaban los principios fundamentales de la integración. Se conjugaban cuestiones de todo tipo, desde la estabilidad administrativa del Estado a la consolidación de un mercado nacional; desde la necesidad de ocupación efectiva del territorio para el ejercicio de la soberanía, hasta dar los pasos necesarios para crear los imaginarios políticos que servirían como formas de cohesión social a la naciente nación. En este proceso se producirá en el espacio del Pacifico sur y las fronteras de Chile con el Perú y Bolivia, la llamada guerra de chile contra la Confederación Perú - boliviana. Este suceso viene a romper momentáneamente la ruta y el espíritu de la ruta emancipadora y a reavivar los primeros signos y síntomas del proto-nacionalismo sudamericano. Mientras en Chile se cerraba la década de 1820, en Perú tampoco se lograba estabilizar el nuevo orden republicano desatándose los mismos conflictos políticos internos que en Chile o en Bolivia. En el caso de Bolivia, el general Andrés de Santa Cruz lograba darle una mayor estabilidad al país y teniendo aliados en el propio Perú, logró formar una confederación en 1836 que incluyó a Perú y Bolivia en una unidad que se proyectaba como poderosa ${ }^{21}$. Ello contribuyó a romper ese espíritu de la ruta de los años 1820. El Perú no había logrado pagar los costos del préstamo chileno de los años 1820 y miraba

\footnotetext{
${ }^{20}$ Idem. p. 101.

${ }^{21} \mathrm{Cid}$, Gabriel. 2011. La guerra contra la federación. Imaginario nacionalista y memoria colectiva en el siglo XIX chileno, Santiago, Ediciones Universidad Diego Portales.
} 
con cierto recelo la insistencia de Chile en que resarciera los costos de la expedición de 1821. Por otra parte, las diferencias entre Valparaíso y el Callo por la exclusividad de ciertas rutas de navegación en el Pacifico y la competencia por los mercados, llevó a que la situación política se deteriora. Valparaíso nuevamente aparecerá siendo el escenario de acopio de recursos navales y de tropas, pero esta vez camino a una guerra no contra tropas extra-americanas sino con los vecinos Perú y Bolivia. El puerto chileno vio partir en 1837 una fuerza expedicionaria compuesta por 2.880 hombres al mando del almirante Manuel Blanco Encalada, quien intentó controlar la situación desde el sur del Perú, siendo derrotado por Santa Cruz y obligado a firmar el tratado de Paucarpata que garantizaba el retiro de las tropas chilenas tanto como el reconocimiento de la Confederación. Como los términos del tratado fueron rechazados por las autoridades de Santiago, la guerra no terminó sino hasta 1839 cuando se producirá la batalla de Yungay, momento en que la derrota de Santa Cruz lo hará huir a Ecuador y la confederación terminará por derruirse ${ }^{22}$. Y esto no será el último acontecimiento relacionado con integración o conflicto que sorprenderá a Valparaíso como testigo y protagonista.

\section{Consideraciones finales}

Hemos analizado el modo histórico con que Valparaíso se ha constituido en uno de los ejes claves a través del cual se coordinó la ruta de O’Higgins - San Martín y luego en lo que caracterizamos como la primera crisis en la idea de integración regional por los hechos de la Guerra contra la Confederación. Hemos valorado el momento en el que el puerto se incardinó en la ruta de O'Higgins - San Martín siendo parte del eje Valparaíso - Santiago y despuntando por su creciente importancia económica, compitiendo con el mismo Santiago. Si proyectamos el crecimiento de Valparaíso en las décadas siguientes y su rol esencial en el crecimiento y consolidación de la República en Chile, lo que quiero subrayar es que desde el punto de vista del análisis historiográfico, en cada uno de los momentos de la ruta - lo previo, el durante y el después - las conexiones con las realidades locales y las realidades más globales no operaban ni como contextos ni como antecedentes sino como constantes que delineaban el horizonte al interior del cual bullía la historia. En esta sinfonía multifónica y policromática, la historia de Valparaíso tiene especificaciones particulares que desde el siglo XVI conectan su devenir a zonas con las cuales formará región y desde esta dinámica será posible descubrir sus ramificaciones que nos llevarán de lo local a lo regional y de ahí a lo global. Para experimentar esta conexión no sólo se requerirá que un día aparezca San Martín viajando desde la pampa para organizar con O'Higgins una escuadra que partiendo desde sus muelles navegue a liberar Perú, sino también el puerto tendría, tal como el Callao, sus propias inquietudes y aspiraciones

\footnotetext{
22 Revisar para todo esto Araya, Eduardo y Sioux, María Luisa. 2008. "Independencia y formaciones nacional”, en Cavieres, Eduardo y Cajías, Fernando, Chile-Bolivia, Bolivia-Chile: 1820-1930. Desarrollos políticos, económicos y culturales, Valparaíso, Ediciones Universitarias de Valparaíso, pp.13 - 46.
} 
interiores que harán ceder las miradas y las superiores intenciones de San Martín y O'Higgins en pos de relaciones afectuosas y permanentes entre ambas naciones. El propio crecimiento germinará en competencias más que en colaboración. Pese a todo, San Martín y O’Higgins siguen mirando el zarpe de la Escuadra Libertadora desde Valparaíso.

\section{Referencias citadas}

Araya, Eduardo y Sioux, María Luisa. 2008. "Independencia y formaciones nacional", en: Cavieres, Eduardo y Cajías, Fernando, Chile-Bolivia, Bolivia-Chile: 1820-1930. Desarrollos políticos, económicos y culturales, Valparaíso, Ediciones Universitarias de Valparaíso.

Barros Arana, Diego. 1892. Historia general de Chile, Tomo XII, Santiago de Chile, Editor Rafael Jover. Campos, Fernando. 1977. Historia constitucional de Chile, Santiago de Chile, Editorial Jurídica.

Cid, Gabriel. 2011. La guerra contra la federación. Imaginario nacionalista y memoria colectiva en el siglo XIX chileno, Santiago de Chile, Ediciones Universidad Diego Portales.

Cristi, Renato y Ruiz Tagle, Pablo. 2006. La república en Chile. Teoría y práctica del constitucionalismo republicano, Santiago, LOM.

Chust, Manuel y Frasquet, Ivana. 2013. Tiempos de revolución. Comprender las independencias iberoamericanas, Madrid, Fundación Mapfre y Editorial Taurus.

Estrada, Baldomero. 2012. Desarrollo empresarial urbano e inmigración europea: españoles en Valparaíso 1880-1940, Universidad Complutense, Madrid, Tesis para optar al grado de Doctor.

Fernández, Sebastián y Vito, Jaime. 2017. "El republicanismo en las Provincias Unidas del Rio de La Palta y Chile en la primera mitad del siglo XIX", en: Cicerchia, Ricardo (comp.), Región y naciones. Instituciones, ciudadanía y performances sociales. Chile y Argentina (siglos XIX-XX). De lo local a lo Global, Buenos Aires, Editorial Prohistoria ediciones y PEI Sur-PUCV, pp. 23-68.

Garreaud, Jacqueline. 1984. "La formación de un mercado de tránsito, Valparaíso: 1817-1848”, en Revista Nueva Historia, Revista de Historia de Chile, Año 3, №11, Londres, FALTA PP.

González I., Marcial. 1871. Las sociedades anónimas. El crédito y la riqueza en Chile, Santiago, Universidad de Chile.

Graham, María. 1822. Diario de su residencia en Chile (1822) y de su viaje a Brasil (1823), Madrid, Ed. América.

Haigh, Samuel. 1917. Viaje a Chile durante la época de la independencia, Santiago de Chile, Colección de autores extranjeros relativos a Chile, Imprenta Universitaria.

Lord Cochrane. 1863. Conde de Dundonald, Memorias, Lima, Imprenta José Masías.

Rivas Inostroza, Fernando. 2016. "El bombardeo de Valparaíso y la conformación de una opinión pública mundial: Un caso inicial de expectación y juicio internacional", Revista de Política Internacional de la Academia Diplomática del Perú Javier Pérez de Cuellar, № 120, abril - junio, Lima, Perú, pp. 95-108. 
Salazar, Gabriel. 2005. Construcción de Estado en Chile (1800-1837), Democracia de los pueblos, militarismo ciudadano, golpismo oligárquico, Santiago de Chile, Editorial sudamericana.

Salinas, René. 1971. "Caracteres Generales de la evolución demográfica de un centro urbano chileno: Valparaíso, 1685-1830", Revista Historia 10, Santiago de Chile, Instituto de Historia Pontificia Universidad Católica de Chile, pp. 177-204.

Véliz, Claudio. 2011. Los dos mundos del Nuevo mundo. Cultura y economía en Angloamérica e Hispanoamérica, Santiago de Chile, Ediciones Tajamar.

Vicuña Mackenna. 1936. Benjamín, Historia de Valparaíso, Obras Completas Volumen III, Tomo I, Santiago de Chile, Universidad de Chile.

Vito, Jaime. 2018. "Problematizando la definición del espacio público moderno: Un acercamiento desde una mirada historiográfica”, en: Cavieres Fernández, Eduardo y Cavieres Figueroa, Eduardo (eds.). Tensiones entre Estado y Ciudadanía. Repensando espacios, significaciones y tiempos, Valparaíso, PEI-SUR- PUCV y CEA Centro de Estudios Avanzados de la Universidad de Playa Ancha, Ediciones de la Editorial de la Universidad de Playa Ancha.

Vito, Jaime y Cavieres, Eduardo. 2020. "Región y nación: Valparaíso- Aconcagua y la configuración de una región en el naciente Chile republicano", en: Cartes Montory, Armando (ed). Región y Nación. La construcción provincial de Chile Siglo XIX, Santiago de Chile, Editorial Universitaria. 